\title{
Carbon Nanotubes Self-Assembled by Amphiphilic Peptide -Helices
}

\author{
I. H. Musselman, ${ }^{*}$ A. B. Dalton, ${ }^{* *}$ A. Ortiz-Acevedo, ${ }^{*}$ J. Razal, ${ }^{* *}$ J. Chen, ${ }^{* * *}$ E. Muñoz, ${ }^{* *}$ \\ R. H. Baughman, ${ }^{* *}$ R. K. Draper, ${ }^{* * * *}$ and G. R. Dieckmann* \\ *Dept. of Chemistry, University of Texas at Dallas, Richardson, TX 75083 \\ **NanoTech Institute, University of Texas at Dallas, Richardson, TX 75083 \\ ***Zyvex Corporation, 1321 North Plano Road, Richardson, TX 75081 \\ ****Dept. of Molecular and Cell Biology, University of Texas at Dallas, Richardson, TX 75083
}

Carbon single-walled nanotubes (SWNTs) are hollow cylinders, each with a single-layered wall of graphite, that have diameters ranging from $\sim 0.5$ to $5 \mathrm{~nm}$ and lengths that exceed $1 \mu \mathrm{m}$ [1]. SWNTs have novel electrical and mechanical properties and are either conducting or semiconducting depending on their diameter and the spiral alignment of the benzene rings along the tube axis. The interest in exploiting the properties of carbon nanotubes in novel electrical and mechanical devices and as components of light and strong materials [2] is tempered by their extreme hydrophobicity and insolubility in most solvents including water. As a result, it is difficult to organize SWNTs into ordered, homogeneous assemblies required for nano-scale devices. We have designed and synthesized a peptide that folds into an amphiphilic -helix and coats carbon nanotubes [3]. The hydrophobic face of the helix interacts noncovalently with the aromatic surface of the carbon nanotubes and the hydrophilic face promotes the self-assembly of coated nanotubes through charged peptide-peptide interactions. Structures of different sizes and shapes are obtained by manipulating solution conditions that affect peptide-peptide interactions.

Nano-1, a 29-residue peptide with the sequence Ac-E(VEAFEKK)(VAAFESK)(VQAFEKK) (VEAFEHG)-CONH 2 , was synthesized using standard solid-phase peptide synthesis methods [3]. Peptide samples were prepared at the desired concentration by diluting a stock amount with deionized water. As-synthesized HiPco SWNTs were purchased from Carbon Nanotechnologies Inc. Peptide/nanotube solutions were prepared by placing $0.15 \mathrm{mg}$ SWNTs on the surface of $3 \mathrm{~mL}$ of peptide solution followed by sonication and centrifugation [3]. The supernatant was used in the experiments. CD spectral measurements were made using an Aviv model 202 circular dichroism spectrometer. Raman spectra were recorded on a Jobin Yvon Horiba high-resolution LabRam Raman microscope system. SEM images of uncoated samples were acquired at $1 \mathrm{keV}$ using a LEO 1530 field-emission scanning electron microscope. TEM images were acquired at $120 \mathrm{keV}$ using a JEOL 2000FX transmission electron microscope.

A model illustrating the potential interactions between nano-1 and a SWNT is provided in Fig. 1A. CD revealed that nano-1 adopts an -helical conformation in aqueous solution. When sonicated in the presence of $100 \mu \mathrm{M}$ aqueous nano-1, the nanotubes dispersed forming homogeneous black solutions that were stable for $>12$ weeks (Fig. 1B). Low-resolution TEM revealed an extensive network of $\sim 10 \mathrm{~nm}$ microfibrils coated with a web-like film of peptide. High-resolution TEM of the microfibrils in the network showed bundles of SWNTs that were highly aligned with the microfibril axis (Fig. 1C). Raman spectroscopy strongly suggested that the peptide intercalated between the nanotubes and that the individual carbon nanotubes were aligned along the fiber axis. Upon dilution with distilled water, small microfibrils $\sim 100 \mathrm{~nm}$ in diameter settled out of the peptide/nanotube solution (Fig. 1D). The strength of charge-charge interactions associated with peptides coating the 
exterior surface of the SWNTs was manipulated by controlling the ionic strength of the aqueous solution through the addition of $\mathrm{NaCl}$. The peptide-coated nanotubes assembled into fibrous structures with diameters of $22 \pm 5$ and $38 \pm 8 \mu \mathrm{m}$ upon addition of 40 (Fig. 1E) and $120 \mathrm{mM} \mathrm{NaCl}$, respectively. The addition of amphiphilic N,N-dimethylformamide (DMF) to a peptide-wrapped nanotube solution modified the structure of the fibrous material by interacting with one or more specific surfaces of the growing crystal. Fig. 1F reveals a ribbon-like structure formed in the presence of $0.0015 \%$ (by volume) DMF. In summary, these data demonstrate that amphiphilic peptides both solubilize carbon nanotubes and control their assembly into various fibrous structures. This structural control of carbon nanotubes by peptides is a first step towards arranging carbon nanotubes into architectures useful for electrical circuits and molecular sensors.

\section{References}

[1] M. S. Dresselhaus et al., Eds. Carbon Nanotubes: Synthesis, Structure, Properties, and Applications, Springer, New York, 2001, Vol. 80.

[2] R. H. Baughman et al., Science 297 (2002) 787.

[3] G. R. Dieckmann et al., J. Am. Chem. Soc. (2003) in press.

[4] We thank Dr. Bog G. Kim for assistance with SEM measurements. This research was supported by The Robert A. Welch Foundation [AT-1326 (IHM), AT-1448 (GRD)], the Texas Advanced Research Program [009741-0026-1999 (GRD)], and a University of Texas at Dallas Special Faculty Development Assignment (RKD).

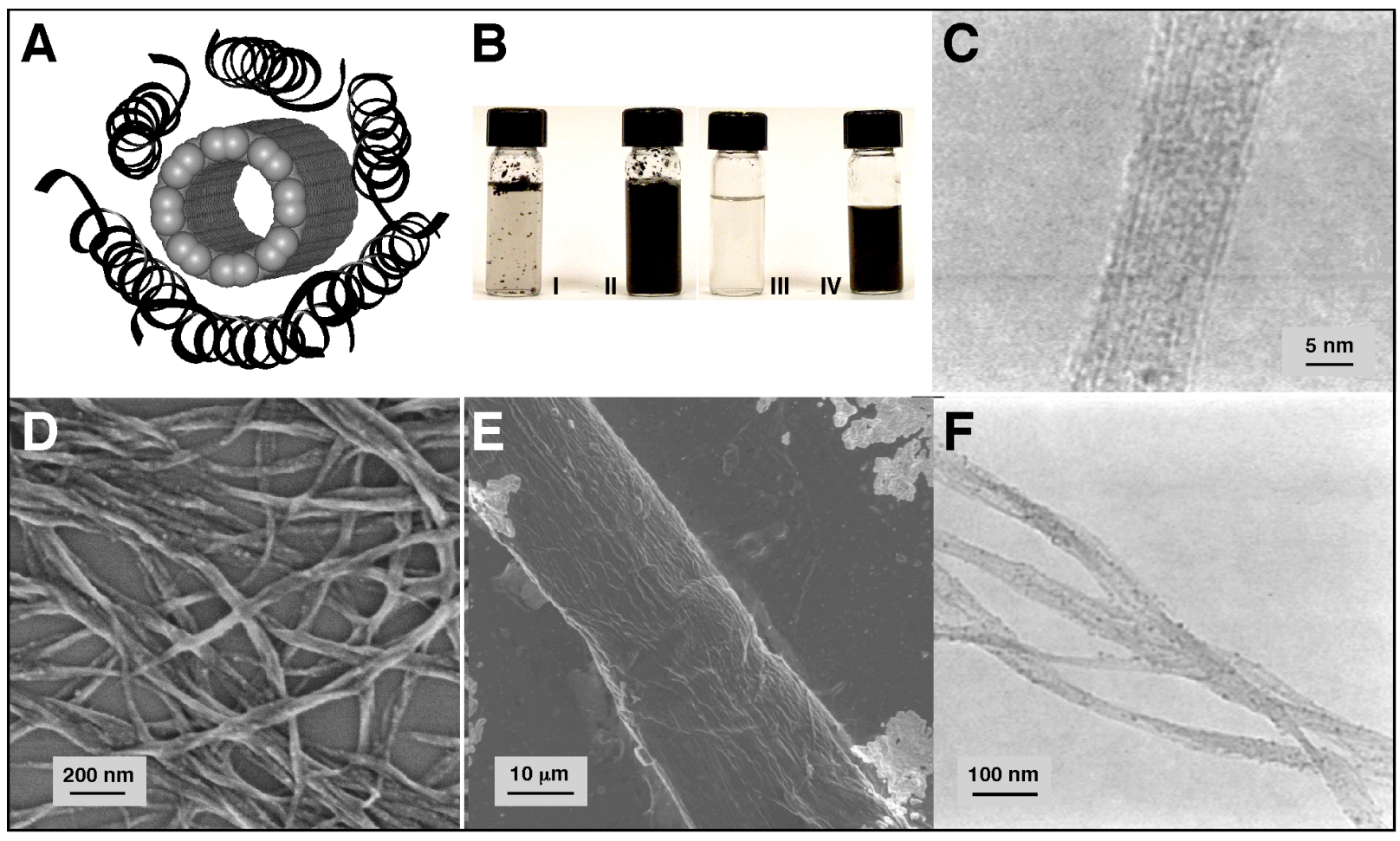

Fig. 1. A. Model of nano-1 -helices and SWNT; B. SWNT solutions with (II,IV) and without (I,III) nano-1 before (I,II) and after (III,IV) filtering; C. SWNT bundles aligned with microfibril axis; D. Microfibrils deposited out of aqueous solution; E. Fibrous structure formed upon addition of $\mathrm{NaCl}$; F. Ribbon structure formed upon addition of DMF. 